\title{
Cinetique De Fermentation De Trois Methodes De Production De Ferments De Racines De Manioc
}

\author{
Abodjo Celah Kakou \\ Zamblé Bi Irié Abel Boli
}

Laboratoire de Biotechnologie et Microbiologie des Aliments, UFR des

Sciences et Technologie des Aliments, Université Nangui Abrogoua,

Abidjan, Côte d'Ivoire

\section{Ollo Kambire}

Département de Biotechnologie et Sciences des Aliments,

Université Peleforo Gon Coulibaly, Côte d'Ivoire

\section{Marina Koussemon \\ Nevry Rose Koffi}

Laboratoire de Biotechnologie et Microbiologie des Aliments, UFR des

Sciences et Technologie des Aliments, Université Nangui Abrogoua,

Abidjan, Côte d'Ivoire

Doi: 10.19044/esj.2017.v13n33p473 URL:http://dx.doi.org/10.19044/esj.2017.v13n33p473

\begin{abstract}
Fermentation plays an important role in the production of several foods. In Côte.d'ivoire, the preparation of several traditional mets of the manioc requires the use of the traditional leavens. Thus, the objective of this study is to compare the evolution of some biochemical and microbiological parameters during the preparation of three types of experimental leavens starting from the roots of varieties bitter and soft of manioc. On the whole, 36 samples of roots of manioc whose 18 of the bitter variety (IAC) and 18 of the soft variety (Bonoua) were the subject of this study. The variety of manioc and the method of acquisition the leavens influence the evolution of the parameters such as the $\mathrm{pH}$, sugars, the growth of the micro-organisms whatever the type of leaven. The leavens of boiled manioc are richer in total sugars while the leavens of believed manioc are it for reducing sugars.
\end{abstract}

Keywords: Leaven, Manioc, Variety, Fermentation.

\section{Resume}

La fermentation joue un rôle important dans la production de plusieurs aliments. En Côte d'Ivoire, la préparation de plusieurs mets traditionnels du manioc exige l'utilisation des ferments traditionnels. Ainsi, 
l'objectif de cette étude est de comparer l'évolution de quelques paramètres biochimiques et microbiologiques au cours de la préparation de trois types de ferments expérimentaux à partir des racines de variétés amère et douce de manioc. Au total 36 échantillons de racines de manioc dont 18 de la variété amère (IAC) et 18 de la variété douce (Bonoua) ont fait l'objet de cette étude. La variété de manioc et la méthode d'obtention des ferments influencent l'évolution des paramètres tels que le $\mathrm{pH}$, les sucres, la croissance des microorganismes quel que soit le type de ferment. Les ferments de manioc bouilli sont plus riches en sucres totaux tandis que les ferments de manioc cru le sont pour les sucres réducteurs.

Mots clés : Ferment, Manioc, Variété, Fermentation.

\section{Introduction}

Le manioc (Manihot esculenta Crantz) est une plante de la famille des Euphorbiaceae à racines tubéreuses des zones tropicales. C'est est une source significative de calories de plus de 500 millions de personnes dans le monde (Fao, 2008), et se présente comme l'une des plantes vivrières les plus importantes par sa production et sa consommation (Yao, 2015a). A ce titre, c'est une plante capable de relayer les cultures vivrières telles que le mil, le maïs et le sorgho (Diallo et al., 2013). Elle est cultivée sur 19,6 millions d'hectares dans le monde pour une production de 252,2 millions de tonnes (MT), et une productivité moyenne de 12,8 MT.

Après le Nigéria, premier pays producteur en Afrique avec 52,4 MT sur 140,4 MT (FAOSTAT, 2011), la production ivoirienne est estimée à plus de 4,5 MT (MINAGRA, 2017). En Côte d'Ivoire, le manioc est la deuxième culture vivrière après l'igname et est utilisé pour la préparation de plusieurs mets traditionnels notamment l'attiéké, le gari, l'agbelima, le tapioca, le fufu, le placali (Yao et al., 2015b). La plupart des mets traditionnels issus du manioc subissent des étapes de transformation au cours desquelles intervient la fermentation.

En Côte d'Ivoire, des ferments traditionnels sont utilisés pour la fermentation de la pâte de manioc en vue de la préparation des mets traditionnels. La production de ces ferments change selon les modes de préparation et les ethnies. La méthode de préparation traditionnelle consiste à fermenter les racines de manioc. L'utilisation de ces ferments traditionnels est spécifique aux régions de production. Chez certaines ethnies notamment, Ebrié et Adjoukrou, les ferments traditionnels sont obtenus par ébullition des racines fraîches de manioc épluchées suivi de leur maintien dans des sachets ou dans des sacs en jute, conditionnés à température ambiante pendant 24 à 72 heures. Par contre, en région Alladjan, les ferments traditionnels sont obtenus avec les racines fraîches de manioc braisées et conservés (Kakou et 
al., 2010). Quant en région Abouré, les ferments traditionnels sont produits avec les racines fraîches de manioc conservé (Tetchi et al., 2012).

Les ferments traditionnels sont colonisés par une multitude de microorganismes qui constitue la source microbienne pendant la fermentation de la pâte manioc (Djeni et al., 2008). Plusieurs études ont identifié des microorganismes tels que les bactéries lactiques, les Bacillus, les coliformes, les levures et moisissures dans la fermentation du manioc (Kakou et al., 2010 ; Akely, 2012 ; Bouatenin et al., 2013).

Compte tenu de la pluralité des microorganismes constitutifs de la flore associée aux ferments traditionnels et de l'effet que pourrait avoir le mode de préparation sur la charge et les souches fermentaires, cette étude a été envisagée afin de suivre l'évolution de quelques paramètres biochimiques et microbiologiques au cours de la préparation de trois types de ferments expérimentaux à partir des racines de variétés amère et douce de manioc.

\section{Materials and Methods}

\section{Matériel d'étude}

Le matériel d'étude est constitué de deux variétés de manioc (Manihot esculenta Crantz) : variété amère IAC (Figure 1A) et variété douce Bonoua (Figure 1B). Les deux variétés de manioc âgées de 8 à 9 mois, ont été récoltées dans un champ monoculture de manioc du village d'Akéikoi, sol de type ferralitique situé dans la commune d'Anyama à $10 \mathrm{~km}$ au nord de la ville Abidjan, Côte d'Ivoire.

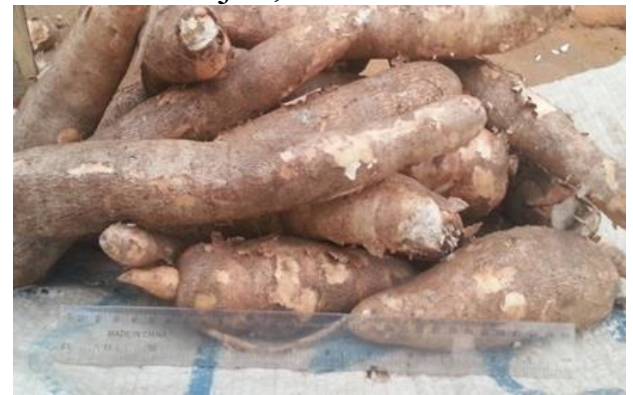

1A : Racines fraiches de manioc (variété amère $I A C$ )

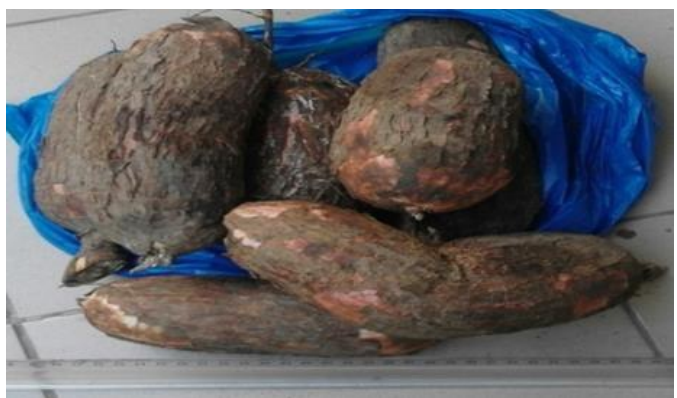

1B : Racines fraiches de manioc (variété douce Bonoua)

Figure 1 : Racines de manioc utilisé pour la préparation des ferments expérimentaux

\section{Collecte des échantillons de racines fraîches de manioc}

Les racines fraîches de deux variétés de manioc (amer et doux) âgées de 8 à 9 mois, ont été collectées dans un même champ décrit comme précédemment en vue de la préparation de ferments expérimentaux. Chaque variété de manioc de $3 \mathrm{~kg}$ environs a été récoltée sur une période de 3 mois à chaque 2 semaines pour permettre une bonne préparation des ferments 
expérimentaux avant les prochaines collectes. Au total, 36 échantillons dont 18 de la variété amère et 18 de la variété douce ont fait l'objet de cette étude. Tous ces échantillons ont été transportés dans des sacs en nylon au laboratoire pour la préparation des différents ferments expérimentaux.

\section{Préparation des ferments expérimentaux}

Les ferments expérimentaux ont été préparés à partir des racines de manioc de la variété amère et douce selon trois méthodes de préparation de ferments traditionnels (Figure 2).

\section{Ferment expérimental obtenu avec le manioc braisé (ferment Alladjan)}

Trois kilogrammes de chacune des deux variétés de racines de manioc frais non épluchés ont été braisés sur du charbon de bois à feu doux pendant 10 à $20 \mathrm{~min}$. Après cuisson et refroidissement à l'air libre, $500 \mathrm{~g}$ constituant un échantillon ont été immédiatement analysés au temps $\mathrm{T}=0$. Cinq autres échantillons de $500 \mathrm{~g}$ de chaque variété ont été mis dans des sachets alimentaires propres, puis conservés dans un carton fermé à la température ambiante $\left(28\right.$ à $\left.32{ }^{\circ} \mathrm{C}\right)$ pour une fermentation. Toutes les 24 heures, un échantillon de ferments de chaque variété est retiré pour les analyses biochimiques et microbiologiques au bout de 96 heures (Figure 2A).

\section{Ferment expérimental obtenu avec le manioc cru (ferment Abouré)}

Trois kilogrammes de chacune des deux variétés de racines de manioc frais ont été lavés avec de l'eau potable, puis subdivisés en dix morceaux de $250 \mathrm{~g}$ pour chaque variété. Deux morceaux de $250 \mathrm{~g}$ de chacune de ces deux variétés constituant un échantillon de chaque variété ont été immédiatement analysés pour le temps $\mathrm{T}=0$. Les huit autres échantillons ont été mis dans des sachets alimentaires propres, puis conservés dans un carton fermé à la température ambiante $\left(28\right.$ à $\left.32^{\circ} \mathrm{C}\right)$ pour une fermentation. Chaque 24 heures, un échantillon de chaque variété est retiré pour les analyses biochimiques et microbiologiques jusqu'à 96 heures (Figure 2B).

\section{Ferment expérimental obtenu avec le manioc bouilli (ferment Ebrié)}

Trois kilogrammes de chacune des deux variétés de manioc frais ont été lavés avec de l'eau potable, épluchés et de nouveau lavés, puis soumis à la cuisson à l'eau bouillante $\left(100^{\circ} \mathrm{C}\right)$ pendant 10 à $20 \mathrm{~min}$. Après cuisson, les racines ont été refroidies à l'air libre, puis un échantillon constitué de $500 \mathrm{~g}$ de chaque variété a été immédiatement analysée au temps $\mathrm{T}=0$. Cinq autres échantillons de $500 \mathrm{~g}$ de chaque variété ont été mis dans des sachets alimentaires propres, puis conservés dans un carton fermé à température 
ambiante $\left(28\right.$ à $\left.32^{\circ} \mathrm{C}\right)$ pour une fermentation. Chaque 24 heures, un échantillon de chaque variété mis en fermentation est retiré pour les analyses biochimiques et microbiologiques durant 96 heures (Figure 2C).

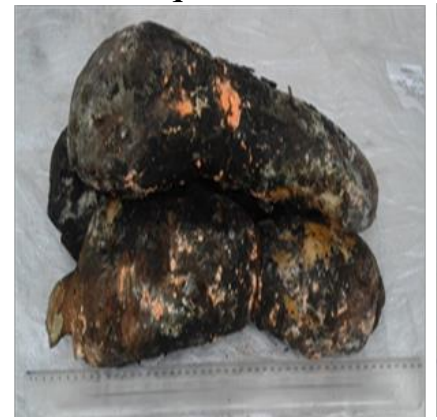

2A : Ferment Alladjan

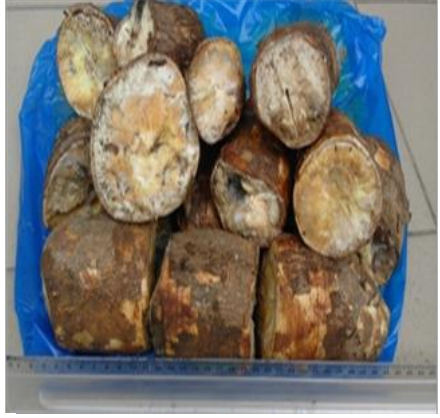

2B : Ferment Abouré

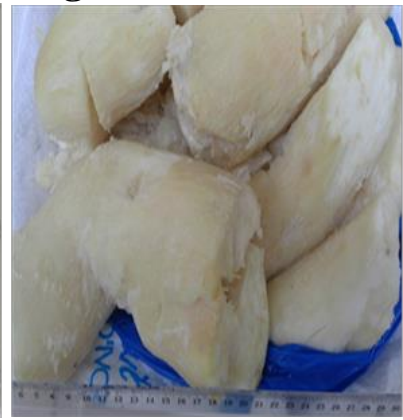

2C : Ferment Ebrié

Figure 2 : Ferments expérimentaux de manioc utilisés en Côte d'Ivoire

\section{Analyse biochimique des ferments expérimentaux}

Le $\mathrm{pH}$ et l'acidité titrable ont été déterminés selon la méthode AOAC (1975). $10 \mathrm{~g}$ de chaque échantillon râpé de chacune des deux variétés de manioc ont été introduits et homogénéisés dans $20 \mathrm{~mL}$ d'eau distillée. Après agitation à l'aide d'un agitateur magnétique (Labovlt, France) et dissolution complète de l'échantillon, le mélange ainsi obtenu constitue la suspension à analyser. Dans cette suspension, a été introduite l'électrode de $\mathrm{pH}$-mètre (P604, Consort, France) et la valeur de $\mathrm{pH}$ déterminée est ainsi affichée sur l'écran du pH-mètre. L'acidité titrable a été déterminée par dissolution de 10 $\mathrm{g}$ de chaque échantillon de chacune des deux variétés de manioc dans de l'eau distillée contenue dans une fiole de $100 \mathrm{~mL}$. Le surnageant a été recueilli par filtration après homogénéisation. Ainsi, $10 \mathrm{~mL}$ de cette solution préalablement étalonnée par de 3 gouttes de phénolphtaléine sont titrés avec une solution de soude de normalité $(0,1 \mathbf{N})$ jusqu'au virage au rose. Les sucres totaux et réducteurs ont été déterminés selon la méthode de Bertrand et Thomas (1910) qui consiste à faire agir les sucres réducteurs sur la liqueur de Fehling en excès. Ainsi, les teneurs en sucres totaux et réducteurs ont été réalisées par dosage au permanganate de potassium $0,1 \mathrm{~N}$.

\section{Analyse microbiologique des ferments expérimentaux}

Pour l'isolation des microorganismes, $10 \mathrm{~g}$ de chaque échantillon de ferments de manioc ont été ajoutés à $90 \mathrm{~mL}$ d'eau peptonée tamponnée stérile dans un sachet Stomacher (AES laboratoire, France). Le mélange a été réalisé par malaxage pour homogénéisation pendant 1 minute. La suspension obtenue a été considérée comme la suspension mère. Ensuite, des dilutions décimales successives ont été effectuées jusqu'à $10^{-6}$ avec $9 \mathrm{~mL}$ d'eau peptonée tamponnée stérile. Le dénombrement des germes aérobies 
mésophiles (GAM) a été effectué selon la norme AFNOR NF V08-051 appliqué à la gélose Plate Count Agar (PCA) dans la masse, celui des coliformes suivant la norme AFNOR FN ISO4832 : 2006 appliqué à la gélose lactosée biliée au cristal violet et au rouge neutre, celui des levures et moisissures selon la norme FN ISO 6611 : 2004 appliqué à la gélose de Sabouraud au Chloramphénicol, celui des bactéries lactiques selon la norme ISO 15214 : 1998 appliqué sur la gélose Man Rogosa Sharpe et celui des Bacillus selon la méthode décrite par Buttiaux et al. (1974) appliqué à la gélose PCA. Les charges microbiennes ont été exprimées en UFC/g (unité formant colonie par gramme).

\section{Resultats}

\section{Evolution des paramètres physico-chimiques au cours de la préparation des ferments traditionnels de manioc}

L'évolution des paramètres biochimiques des ferments issus des variétés amère $(I A C)$ et douce (Bonoua) de manioc au cours de 96 heures de la fermentation est illustrée par la figure 3. Le $\mathrm{pH}$ diminue progressivement au cours de la fermentation quels que soit la variété et le type de ferment de manioc. Dans les différents ferments de manioc, les valeurs de $\mathrm{pH}$ sont passées de 7,27 à 4,3 pour la variété amère et de 7,33 à 4,6 pour la variété douce au niveau des ferments de manioc bouilli, de 6,08 et de 6,89 à 3,75 et 3,13 respectivement pour les variétés amère et douce dans le cas des ferments de manioc cru et de 7,01 (variété douce) et de 7,16 (variété amère) à 4,47 et 3,83 respectivement au niveau des ferments de manioc braisé (Figure 3A). En ce qui concerne l'acidité titrable, les taux ont varié de 0,02 à $0,12 \%$ (variété amère) et de 0,02 à $0,09 \%$ (variété douce) pour les ferments de manioc bouilli, de $0,02 \%$ (variété amère) et de $0,026 \%$ (variété douce) à $0,08 \%$ et $0,092 \%$ respectivement pour les ferments de manioc cru et de 0,027 à $0,11 \%$ pour la variété douce et de 0,025 à $0,82 \%$ pour la variété amère dans le cas des ferments de manioc braisé (Figure 3B). Au niveau des sucres totaux, une diminution progressive a été observée pour tous les types de ferments quelle que soit la variété de manioc. Des taux allant de 2,4 à 1,1 $\mathrm{mg} / \mathrm{g}$ (variété amère) et de 2,7 à $1,2 \mathrm{mg} / \mathrm{g}$ (variété douce) pour les ferments de manioc bouilli, de 1,3 à $0,82 \mathrm{mg} / \mathrm{g}$ de matière fraîche et de 2,7 à $1,2 \mathrm{mg} / \mathrm{g}$ de matière fraîche respectivement pour la variété amère et la variété douce des ferments de manioc cru et, de 1,3 à $1,07 \mathrm{mg} / \mathrm{g}$ de matière (variété amère) et de 2,2 à $0,8 \mathrm{mg} / \mathrm{g}$ de matière (variété douce) pour les ferments de manioc braisé (Figure 3C). Pour les sucres réducteurs, une diminution progressive a été également observée quels que soient le type de ferment et la variété de manioc. Des taux diminuant progressivement de 1,1 à $0,4 \mathrm{mg} / \mathrm{g}$ de matière fraîche (variété amère) et de 1,4 à $0,5 \mathrm{mg} / \mathrm{g}$ de matière fraîche (variété douce) dans le cas des ferments de manioc bouilli, de 1,62 à $0,9 \mathrm{mg} / \mathrm{g}$ de 
matière fraîche (variété amère) et de 1,51 à $0,65 \mathrm{mg} / \mathrm{g}$ (variété douce) dans le cas des ferments de manioc cru et, de $1,22 \mathrm{mg} / \mathrm{g}$ de matière fraîche (variété amère) et $1,4 \mathrm{mg} / \mathrm{g}$ de matière fraîche (variété douce) à $0,7 \mathrm{mg} / \mathrm{g}$ et $0,6 \mathrm{mg} / \mathrm{g}$ respectivement dans le cas des ferments de manioc braisé (Figure 3D).
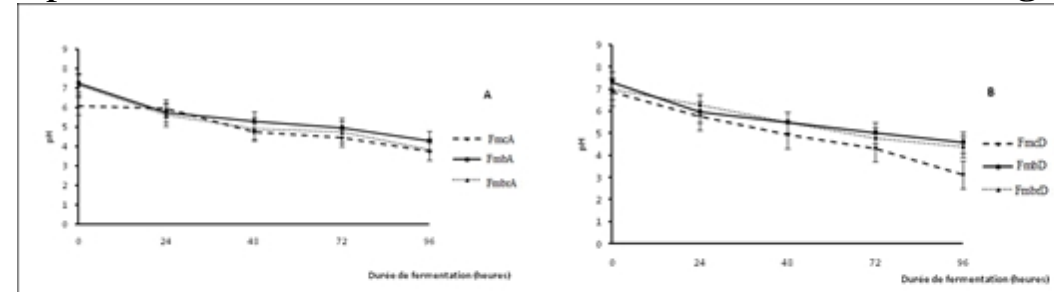

3-A : érolution du $\mathrm{pH}$
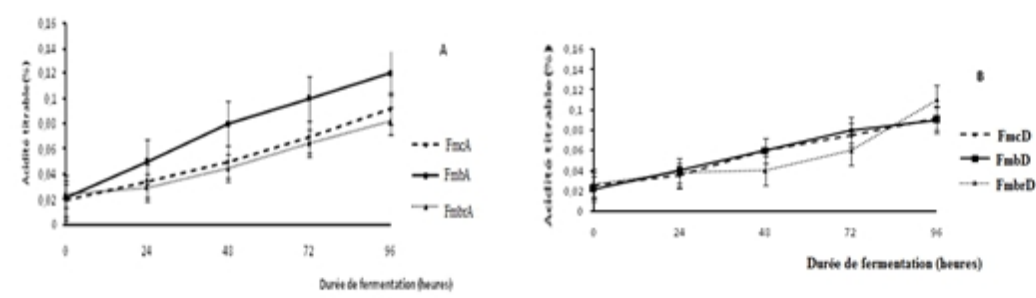

3-B : évolution d'acidité titrable
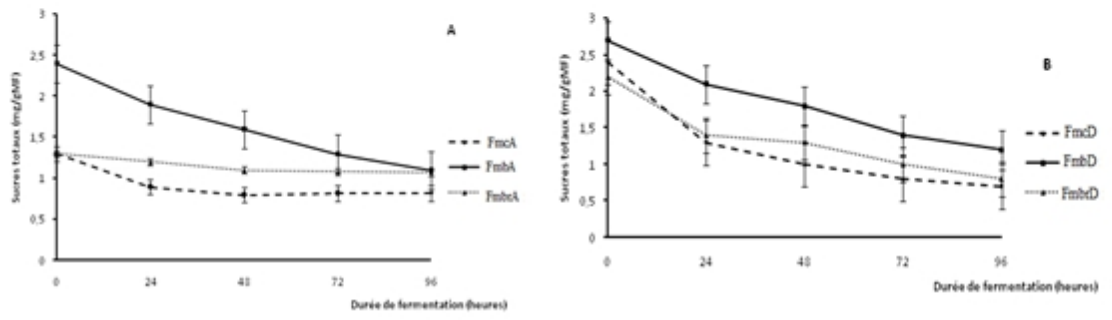

3-C: érolution des sucres totaux
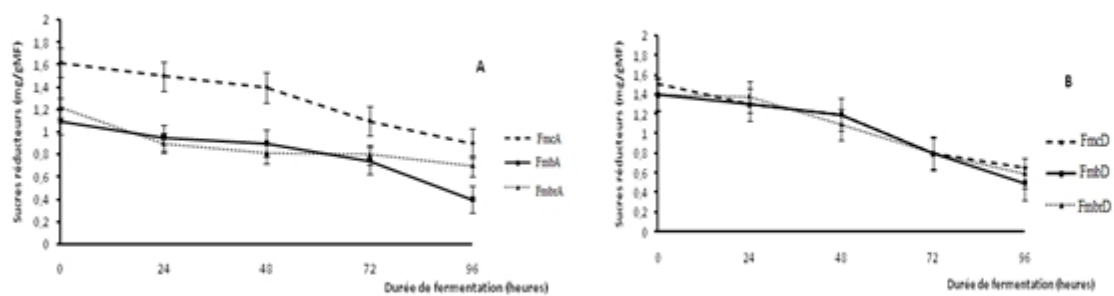

3-D : évolution des sucres réducteurs

Figure 3 : Evolution des paramètres biochimiques des ferments de manioc des variétés amère $(\mathrm{A})$ et douce $(\mathrm{B})$

FmcA : ferment de manioc cru amer ; FmbA : ferment de manioc bouilli amer; FmbrA : ferment de manioc braisé amer ; FmcD : ferment de manioc cru doux ; FmbD : ferment de manioc bouilli doux ; FmbrD : ferment de manioc braisé doux 


\section{Evolution des paramètres microbiologiques au cours de la préparation des ferments traditionnels de manioc}

Les charges microbiennes des ferments issus des variétés amère et douce de manioc au cours de 96 heures de la fermentation sont données dans les tableaux 1 et 2. Il s'agit des Germes aérobies mésophiles (GAM), des bactéries lactiques, des coliformes thermotolérants, des coliformes totaux, des Bacillus, des levures et moisissures. Au niveau de la variété amère, les plus importantes charges dans les trois types de ferment ont été obtenues avec les GAM soit 9,4 $\pm 0,42 \log$ (UFC/g) dans le ferment de manioc braisé, suivies de celles des bactéries lactiques et des Bacillus soient respectivement $6,37 \pm 0,17 \log (\mathrm{UFC} / \mathrm{g})$ et 6,21 $\pm 0,12 \log (\mathrm{UFC} / \mathrm{g})$ obtenues dans les ferments de manioc bouilli. Les coliformes thermotolérants et les levures et moisissures étaient moins représentés dans les ferments avec 3,2 $\pm 0,08$ log (UFC/g) obtenues respectivement dans les maniocs bouilli et cru. Toutefois, une différence significative $(\mathrm{P}<0,05)$ a été observée entre les différentes charges microbiennes au niveau de chaque type de ferment.

Pour ce qui est de la variété douce, la charge des GAM avec des moyennes de 9,52 $\pm 0,49 \log (\mathrm{UFC} / \mathrm{g}$ ) a été importante dans les trois types de ferment, suivis des Bacillus, soit 6,54 $\pm 0,05 \log (\mathrm{UFC} / \mathrm{g})$, des bactéries lactiques et des coliformes totaux avec des charges similaires de 5,93 $\pm 0,03$ $\log (\mathrm{UFC} / \mathrm{g})$ pour la variété douce. Le ferment de manioc braisé était chargé par les GAM tandis que ceux du manioc bouilli était dominé par les Bacillus et du manioc cru par les bactéries lactiques. La plus petite charge microbienne, soit 2,01 $\pm 0,12 \log (\mathrm{UFC} / \mathrm{g}$ ) a été obtenue avec les levures et moisissures dans le ferment de manioc braisé. Une différence significative $(\mathrm{P}<0,05)$ a été observée entre les différentes charges microbiennes au niveau de chaque type de ferment excepté les bactéries lactiques et les coliformes totaux.

Tableau 1 : Charges microbiennes des ferments de la variété amère de manioc

\begin{tabular}{|c|c|c|c|c|c|c|}
\hline \multirow[b]{2}{*}{$\begin{array}{l}\text { Type de } \\
\text { ferment }\end{array}$} & \multicolumn{6}{|c|}{ Charges des microorganismes $(\log 10 \mathrm{UFC} / \mathrm{g})$} \\
\hline & GAM & $\begin{array}{l}\text { Bactéries } \\
\text { lactiques }\end{array}$ & $\begin{array}{c}\text { Coliformes } \\
\text { totaux }\end{array}$ & $\begin{array}{c}\text { Coliformes } \\
\text { thermotolérants }\end{array}$ & Bacillus & $\begin{array}{c}\text { Levures et } \\
\text { Moisissures }\end{array}$ \\
\hline Bouilli & $7,76 \pm 0,42^{\mathrm{a}}$ & $6,37 \pm 0,17^{\mathrm{b}}$ & $5,9 \pm 0,16^{\mathrm{b}}$ & $3,2 \pm 0,52^{\mathrm{a}}$ & $6,21 \pm 0,12^{\mathrm{b}}$ & $3,41 \pm 0,09^{\mathrm{b}}$ \\
\hline Cru & $7,9 \pm 0,45^{\mathrm{a}}$ & $5,67 \pm 0,33^{\mathrm{a}}$ & $5,14 \pm 0,37^{\mathrm{a}}$ & $3,68 \pm 0,4^{\mathrm{ab}}$ & $5,47 \pm 0,27^{\mathrm{a}}$ & $3,2 \pm 0,08^{\mathrm{ab}}$ \\
\hline Braisé & $9,4 \pm 0,42^{b}$ & $5,17 \pm 0,13^{\mathrm{a}}$ & $5,8 \pm 0,09^{\mathrm{b}}$ & $4,3 \pm 0,17^{b}$ & $5,67 \pm 0,05^{\mathrm{a}}$ & $3,05 \pm 0,02^{\mathrm{a}}$ \\
\hline
\end{tabular}

En colonne, les moyennes affectées d'une même lettre ne sont pas significativement différentes au seuil de 5\% 
Tableau 2 : Charges microbiennes des ferments de la variété douce de manioc

\begin{tabular}{ccccccc}
\hline & \multicolumn{5}{c}{ Charges des microorganismes (log10 UFC/g) } \\
\cline { 2 - 7 } $\begin{array}{c}\text { Types de } \\
\text { ferment }\end{array}$ & GAM & $\begin{array}{c}\text { Bactéries } \\
\text { lactiques }\end{array}$ & $\begin{array}{c}\text { Coliformes } \\
\text { totaux }\end{array}$ & $\begin{array}{c}\text { Coliformes } \\
\text { thermotolérants }\end{array}$ & Bacillus & $\begin{array}{c}\text { Levures et } \\
\text { Moisissures }\end{array}$ \\
\hline Bouilli & $7,44 \pm 0,3^{\mathrm{a}}$ & $5,8 \pm 0,11^{\mathrm{a}}$ & $5,8 \pm 0,11^{\mathrm{a}}$ & $4,12 \pm 0,26^{\mathrm{a}}$ & $6,54 \pm 0,05^{\mathrm{b}}$ & $2,22 \pm 0,14^{\mathrm{a}}$ \\
Cru & $8,9 \pm 0,15^{\mathrm{a}}$ & $5,93 \pm 0,03^{\mathrm{a}}$ & $5,93 \pm 0,03^{\mathrm{a}}$ & $4,7 \pm 0,34^{\mathrm{ab}}$ & $6,33 \pm 0,27^{\mathrm{b}}$ & $3,33 \pm 0,22^{\mathrm{b}}$ \\
Braisé & $9,52 \pm 0,49^{\mathrm{b}}$ & $5,76 \pm 0,16^{\mathrm{a}}$ & $5,76 \pm 0,16^{\mathrm{a}}$ & $5,2 \pm 0,09^{\mathrm{b}}$ & $5,67 \pm 0,07^{\mathrm{a}}$ & $2,01 \pm 0,12^{\mathrm{a}}$ \\
\hline
\end{tabular}

En colonne, les moyennes affectées d'une même lettre ne sont pas significativement différentes au seuil de 5\%

La répartition des charges microbiennes totales des ferments de manioc selon la variété est présentée dans le tableau 3. La charge microbienne la plus importante notamment 8,62 $\pm 0,97 \log (\mathrm{UFC} / \mathrm{g})$ a été obtenue avec les GAM dans les ferments de la variété douce de manioc, tandis que la plus petite charge, notamment celle des levures et moisissures, soit 3,22 $\pm 0,19 \log (\mathrm{UFC} / \mathrm{g})$ a été obtenue avec les ferments de la variété amère de manioc. Toutefois, une différence significative $(\mathrm{P}<0,05)$ a été observée entre les différentes charges microbiennes au niveau des deux variétés de manioc, hormis les GAM et les bactéries lactiques.

Tableau 3 : Charges microbiennes totales des ferments de manioc selon la variété

\begin{tabular}{ccccccc}
\hline \multirow{2}{*}{$\begin{array}{c}\text { Variétés } \\
\text { de manioc }\end{array}$} & GAM & $\begin{array}{c}\text { Bactéries } \\
\text { lactiques }\end{array}$ & $\begin{array}{c}\text { Coliformes } \\
\text { totaux }\end{array}$ & $\begin{array}{c}\text { Coliformes } \\
\text { thermotolérants }\end{array}$ & Bacillus & $\begin{array}{c}\text { Levures et } \\
\text { moisissures }\end{array}$ \\
\hline Amère & $8,35 \pm 0,35^{\mathrm{a}}$ & $5,74 \pm 0,55^{\mathrm{a}}$ & $5,61 \pm 0,41^{\mathrm{a}}$ & $3,72 \pm 0,58^{\mathrm{a}}$ & $5,78 \pm 0,36^{\mathrm{a}}$ & $3,22 \pm 0,19^{\mathrm{a}}$ \\
Douce & $8,62 \pm 0,97^{\mathrm{a}}$ & $5,83 \pm 0,12^{\mathrm{a}}$ & $6,04 \pm 0,25^{\mathrm{b}}$ & $4,67 \pm 0,51^{\mathrm{b}}$ & $6,18 \pm 0,42^{\mathrm{b}}$ & $2,52 \pm 0,63^{\mathrm{b}}$ \\
\hline
\end{tabular}

En colonne, les moyennes affectées d'une même lettre ne sont pas significativement différentes au seuil de 5\%

\section{Discussion}

Cette étude a pour but de suivre les paramètres biochimiques et microbiologiques afin de déterminer la durée maximale de préparation des différents types de ferments expérimentaux. Le $\mathrm{pH}$ est un facteur important au cours de la fermentation et de la croissance microbienne. Dans cette étude, le $\mathrm{pH}$ diminue dans les racines de manioc pendant la fermentation quel que soit le type de ferment et la variété de manioc. La diminution progressive du $\mathrm{pH}$ pourrait être due à l'activité des bactéries lactiques. En effet, selon les travaux de Coulin et al. (2006) et Djoulde et al. (2015), les bactéries lactiques qui constituent la microflore dominante du ferment de manioc produisent des acides organiques qui acidifient le milieu de fermentation. Ce qui induit une baisse du $\mathrm{pH}$ du milieu. Inversement au $\mathrm{pH}$, l'acidité titrable connaît une augmentation au cours de la fermentation. L'augmentation de l'acidité titrable au cours de la fermentation des deux variétés de manioc pourrait être liée à l'accumulation des acides organiques 
notamment, les acides lactiques et acétiques produits par les bactéries et les levures comme l'ont rapporté Kobawila et al. (2005) et Oduah et al. (2015).

Les teneurs en sucres totaux et réducteurs diminuent au cours de la fermentation quels que soient le type de ferment et la variété de manioc utilisée. Ce résultat pourrait s'expliquer par l'hydrolyse des sucres contenus dans les racines de manioc en acides organiques au cours du métabolisme fermentaire, sous l'action des enzymes microbiennes. Ces résultats pourraient aussi s'expliquer par l'assimilation des sucres simples après hydrolyse comme nutriments par les microorganismes. En effet, selon Panda et al. (2007), une part substantielle des sucres est utilisée par les microorganismes présents dans le milieu de fermentation. Par ailleurs, Embaby et Abdel-Galil, (2006) et Kakde et Chavan (2011), ont rapporté que les microorganismes sont responsables de l'épuisement des sucres dans les aliments au cours de leur croissance. Ces résultats corroborent ceux des travaux de Soro et al. (2013) et Djoulde et al. (2015) qui ont rapporté la diminution du taux de sucres au cours de la fermentation du manioc.

Cette étude a aussi montré l'influence de la variété et du type de ferments sur les charges moyennes de chaque groupe microbien considéré. La croissance maximale des germes aérobies mésophiles et des coliformes au cours de la préparation des différents types de ferment de manioc a été observée pendant les premières 48 ou 72 heures de fermentation selon le cas. La présence des coliformes en tant que témoins de contamination pourrait impliquer celle de pathogènes. En effet, ces pathogènes pourraient provenir soit du matériel de travail, soit de l'environnement ambiant après cuisson, ou bien soit du milieu de conservation des ferments. Par ailleurs, selon Assanvo et al. (2002) et Achi et Akomas (2006), la présence de coliformes dans les ferments de manioc serait due à des mauvaises qualités hygiéniques au cours de la préparation de des ferments notamment les sacs utilisés, l'eau et hygiène corporelle. Par contre, la baisse de croissance observée par la suite pourrait s'expliquer par l'activité des bactéries lactiques qui jouent un rôle majeur dans la fermentation du manioc. En effet, ces bactéries en produisant des acides organiques pourraient jouer un rôle déterminant dans l'amélioration de la qualité hygiénique du manioc. L'effet inhibiteur des acides lactique et acétique lié à leurs formes dissociées, leur permet d'entrer dans les cellules bactériennes où ils s'ionisent et s'accumulent, provoquant un abaissement interne du $\mathrm{pH}$ et le blocage de mécanismes de transport. L'inhibition des pathogènes par les bactéries lactiques est due au fait que les bactéries lactiques ne possèdent pas de catalase et en présence de l'air, leur métabolisme conduit à une accumulation de peroxyde d'hydrogène $\left(\mathrm{H}_{2} \mathrm{O}_{2}\right)$, autre antiseptique puissant contre les germes pathogènes (Djoulde et al., 2015). Aussi, la baisse de croissance des GAM et des coliformes pourrait être liée aux bactériocines, des toxines 
synthétisées par les bactéries lactiques. En effet, selon Guarner et al. (2005) et Djoulde et al. (2015), les bactéries lactiques synthétisent des bactériocines qui seraient actives contre les germes pathogènes au cours de la fermentation.

Les bactéries lactiques présentent des charges élevées au cours des 72 premières heures de fermentation dans les différents types de ferment quelle que soit la variété de manioc utilisée. Ce résultat pourrait s'expliquer par un nombre élevé de nutriments disponibles dans les racines de manioc pour la croissance des microorganismes. En effet, selon Assanvo et al. (2002), le glucose contenu dans le manioc constitue la substance de base pour la nutrition et la croissance des bactéries lactiques au cours de la fermentation. Le manioc étant pourvu en glucose, après l'hydrolyse de l'amidon par les Bacillus, il devient un substrat favorable à la multiplication des microorganismes. Toutefois, les ferments issus de la variété douce renferment un nombre relativement élevé de bactéries lactiques que ceux de la variété amère de manioc. Ce résultat pourrait s'expliquer par le fait que la variété douce de manioc utilisé au cours de la fermentation serait plus favorable au développement des bactéries lactiques, plus aptes à se développer en milieu acide compte tenu de leur caractère acidophile. La diminution de la charge des bactéries lactiques au-delà de 72 heures pourrait être due soit à l'insuffisance de sucres simples permettant leur croissance, soit à la compétition bactérienne.

La prédominance de Bacillus au cours de la fermentation des deux variétés de manioc pourrait s'expliquer par le fait que ce sont l'un des principaux microorganismes responsables de la fermentation du manioc et participent à la dégradation tissulaire du manioc par la production d'enzymes comme les polygalacturonases, les pectines estérases, les cellulases et les amylases qu'elles libèrent (Ouattara et al., 2008 ; Ehon et al., 2015). De façon générale, les ferments de manioc bouilli et braisé sont plus riches en Bacillus que le ferment de manioc cru, et ceci, quelle que soit la variété de manioc utilisée. Ce résultat pourrait être dû au mode de préparation des ferments de manioc bouilli et braisé qui comporte une phase de cuisson. En effet, selon Akely et al. (2016), la chaleur gélatinise l'amidon ou l'hydrolyse partiellement. Cette hydrolyse conduit à la production de sucres simples qui constituent la source de carbone principale des Bacillus et bien d'autres microorganismes. Aussi, le traitement thermique subi par ces deux ferments sus cités permet-elle d'accroître leur teneur en glucides utilisés comme source de carbone par les Bacillus. Selon Ayoola et al. (2012), la teneur en glucides est plus élevée dans les aliments cuits compte tenu de leur soumission à un traitement thermique. Par ailleurs, Fagbohun et al. (2011) et Ameer et al. (2013) ont rapporté que les glucides sont utilisés comme nutriments favorables à la croissance des microorganismes. Or, le ferment de 
manioc cru est un ferment qui ne aucun traitement thermique au cours de sa préparation.

La présence de levures et moisissures tout au long de la fermentation dans ces deux variétés de manioc serait due au fait qu'elles participent à la fermentation des racines de manioc bien qu'étant en nombre réduit par rapport aux autres microorganismes déterminés. En effet, selon Brauman et al. (1995), les levures métabolisent les hydrates de carbone, notamment le glucose et le fructose pour la production d'acides organiques. La prolifération de ces champignons pourrait être liée à la disponibilité d'hydrate de carbone dans le milieu. En effet, selon Oduah et al. (2015), les hydrates de carbone sont largement utilisés comme source nutritive par les microorganismes au cours de la fermentation des racines de manioc.

\section{Conclusion}

$\mathrm{Au}$ terme de cette étude, il ressort que la variété de manioc et la méthode d'obtention des ferments influencent l'évolution des paramètres biochimiques tels que le $\mathrm{pH}$, les sucres, la croissance des microorganismes quel que soit le type de ferment. Les ferments de manioc bouilli sont plus riches en sucres totaux tandis que les ferments de manioc cru le sont pour les sucres réducteurs. Les ferments de manioc bouilli de la variété douce sont plus riches en micro-organismes fermentaires avec une prédominance des Bacillus dans la variété douce.

\section{References:}

1. Achi O. K. and Akomas N. S., 2006. Comparative Assessment of Fermentation Techniques in the Processing of Fufu, a Traditional Fermented Cassava Product. Pakistan Journal of Nutrition, 5 (3): 224-229.

2. Akely P. M. T., Djina Y., Konan B. R., Irie K., Kouame L. P. and Amani n G., 2016. Study of Varietal Influence Post Conservation on Biochemical and Sensory Qualities of Attiéké and Boiled Cassava (Manihot esculenta Crantz).Agricultural Sciences, 7 : 127-136.

3. Akely P. M. T., 2012. Influence de la fermentation contrôlée du pressage et de la granulation mécanisés du manioc (Manihot esculenta, Crantz) râpé sur les caractéristiques physico-chimiques et sensorielles de l'attiéké. Thèse unique, $\mathrm{N}^{\circ} 183$, UFR/STA, UNA, Abidjan, Côte d'Ivoire. 150p.

4. Ameer J. B., Venudevan B. and Jayanthi B., 2013. Storage Fungi in groundnut and the associate seed quality deterioration.Plant Pathology Journal, 12 (3): 127-134.

5. AOAC., 1995. Official methods of Analysis of AOAC International, 16th ed. AOAC International Arlington, VA, 250 p. 
6. Assanvo J. B., Agbo G. N., Belli Y. E. N., Coulin P. et Fara Z., 2002. La microflore du ferment de manioc pour la production de l'attiéké adjoukrou (Côte d'Ivoire). LBSA (université de Cocody, Côte d'Ivoire), CSRS (Abidjan), ETHZ (Zurich). Bioterre, revue. Internationale des sciences de la vie et de la terre, $n^{\circ}$ spécial, 286299.

7. Ayoola P. B., Adeyeye A. and OnawumI O. O., 2012. Chemical evaluation of food value of groundnut (Arachi hypogaea) seeds. American Journal of Food and Nutrition, 2 (3): 55-57.

8. Bertrand $\mathrm{G}$ et Thomas P., 1910. Guide pour les manipulations de chimie biologie. Dunod, Paris.

9. Bouatenin J. P. K. M., Djeni T. N., Ouassa T., Zinieu E., Menan H. and Dje K. M., 2013. Characterization and Enzyme Activities of Microorganisms from a Traditional Cassava Starter Used for the Production of Adjoukrou Attieke (Cote d'Ivoire). Journal of Food Technology, 11 (1): 4-13

10. Brauman A., Keleke S., Mavoungou O., Ampe F. et Miambi E., 1995. Étude d'une fermentation lactique traditionnelle des racines de manioc en Afrique centrale (Congo), in: transformation alimentaire du manioc, Agbor E., Brauman A., Griffon D. et Treche S., éd ORSTON, Paris, France, 287- 305.

11. Buttiaux R., Beerens H. et Taquet A., 1974. Manuels de techniques bactériologiques, $4^{\text {ème }}$ ed. Flamarion, Paris, 70p.

12. Coulin P., Farah Z., Assanvo J., Spillman H. and Puhan Z., 2006. Characterisation of the microflora of attiéké, a fermented cassava product during traditional small-scale production. International Journal of Food Microbiology, 106 : 131-136.

13. Diallo Y., Gueye M. T., Sakho M., Darboux P. G., Kane A., Barthelemy J-P. et Lognay G., 2013. Importance nutritionnelle du manioc et perspectives pour l'alimentation de base au Sénégal (synthèse bibliographique). Biotechnology Agronomique of. Society and.Environment, 17 (4): 634-643.

14. Djeni N. T., N'guesan K. F., Dadie A. T. and Dje K. M., 2008. Impact of different levels of a traditional starter on the fermentation of cassava dough for Attiéké production. Food, 2 (2): 145-151.

15. Djoulde R. D., Essia J. J. N. and Etoa F. X., 2015. Amélioration du rouissage du manioc par utilisation d'un starter microbien de trois souches. International Journal of Innovation and Scientific Research, 14 (2): 268-277.

16. Ehon A. F., Krabi R. E., Assamoi A. A. and Niamke S. L., 2015. Preliminary technological properties assessment of Bacillus spp. 
Isolated from traditional cassava starters used for attieke production. European Scientific Journal, 11 (9): 177-187.

17. Embaby E. M. and Abdel-Galil M. M., 2006. Seed borne fungi and mycotoxins associated with some legume seeds in Egypt. Journal Applied Sciences Research, 2 : 1064-1071.

18. Fagbohun E. D., Lawal O. U. and Hassan O. A., 2011. The chemical composition and mycoflora of sundried shelled melon seeds (Citrullus vulgaris) during storage. International Journal Research of Microbiology, 2 (8): 330-314.

19. FAO, 2011. FAOSTAT. http://faostat.fao.org/

20. FAO, 2008. The impact of HIV/AIDS on the agricultural sector. Corporate Document Repository, http://www.fao.org/docrep/005/Y4636E/y4636e05.htm.

21. Guarner F., Perdigon G., Corthier G., Salminen S., Koletzko B. and Morelli L., 2005. Should yoghurt cultures be considered probiotic. British Journal of Nutrition, 93 (6): 783-786.

22. Kakde R. B. and Chavan A. M., 2011. Extracellular lipase enzyme production by seed-borne fungi under the influenc of physical facyors. International Journal Biology, 3: 94-100.

23. Kakou A.C., Tagro G., Kambire O., Kouame F., Koffi N. and Koussemon M., 2010. Biochemical and microbial changes during traditional spontaneous lactic acid fermentation process using two varieties of cassava for production of a " Alladjan" starter. International Food Research Journal, 17: 563-573.

24. Kobawila S. C., Louembe D., Keleke S., Hounhouigan J. and Gamba C., 2005. Reduction of the cyanide content during fermentation of cassava root sandleaves to produce bikedi and ntobambodi, two food products from Congo. African Journal of Biotechnologies, 4: 689696.

25. MINAGRI, 2017. Ministère de l'Agriculture (Côte d'Ivoire). Rapporte 1'Agence de Presse Africaine (APA) Annuaire des Statistiques Agricoles 2010-2012. Edition 2012, 1p.

26. Oduah N. O., Adepoju P. A., Longe O., Elemo G. N. and Oke O. V., 2015. Effects Of Fermentation On The Quality And Composition Of Cassava Mash (Gari). International Journal of Food Nutrition and Safety, 6 (1): 30-41.

27. Ouattara H. G., Koffi B. L., Karou G.T., Sangare A., Niamke S. L. and Diopoh J.K., 2008. Implication of Bacillus sp. in the production of pectinolytic enzymes during cocoa fermentation.World Journal Microbiology Biotechnology, 24 : 1753-1760. 
28. Panda S. H., Parmanick M. and Ray R. C., 2007. Lactic acid fermentation of sweetpotato (Ipomoeabatatas L.) into pickles. Journal of Food Procces, 31 : 83-101.

29. Soro S., Konan G., Elleingand E., N'guessan D. and Koffi E., 2013. Formulation d'aliments infantiles à base de farines d'igname enrichies au soja. African Journal of Food, Agriculture, Nutrition and Developpement, $13: 8313-8339$.

30. Tetchi F. A., Solomen O.W., Kakou A. C. and Amani N. G., 2012. Effect of cassava variety and fermentation time on biochemical and microbiological characteristics of raw artisanal starter for "attiéké" production. Innovative Romanian Food Biotechnology, 10 : 40-47.

31. Yao A. K., Koffi D. M., Blei S. H., Irié Z. B. et Niamké S. L., 2015a. Nouvelle technique de transformation de la pulpe de manioc (Maniot esculenta CRANTZ) sous forme de granulés conservables sur une longue période. European Scientific Journal, 11 (24): 415-425.

32. Yao A. K., Koffi D. M., Blei S. H., Irié Z. B. and Niamké S. L., 2015b. Propriétés biochimiques et organoleptiques de trois mets traditionnels ivoiriens (attiéké, placali, attoukpou) à base de granulés de manioc natifs. Int. J. Biol. Chem. Sci., 9 (3): 1341-1353. 